\title{
Relation Entre Les Facteurs Socio-Économiques Et L’anémie Au Cours De La Grossesse (Nouakchott - Mauritanie)
}

\author{
Setty Sass \\ Mariem Sidi Mohamed
}

Laboratoire de Biochimie, Biotechnologie, Environnement et Sante. Université Ibn Tofail. Faculté de Sciences. Département Sciences de la Vie.

Kénitra. Maroc

\section{Ahmed Zein}

Gynécologue obstétrique, Université des Sciences de technologie et de Médecine. Mauritanie

\section{Ahmed Elberae}

Institut National de Recherches en Santé Publique(INRSP). Mauritanie

\section{Abderrazzak Khadmaoui}

Laboratoire de génétique et biométrie, Université Ibn Tofail. Faculté de Sciences. Département Sciences de la Vie. Kénitra. Maroc.

\section{Soad Khllayoun}

\section{Aicha Lrhorfi}

\section{Rachid Bengueddour}

Laboratoire de Biochimie, Biotechnologie, Environnement et Sante. Université Ibn Tofail. Faculté de Sciences. Département Sciences de la Vie. Kénitra. Maroc.

\begin{abstract}
Anemia is one of the most common public health problems in the world, especially among pregnant women. The objective of our study is to describe the socio-demographic and epidemiological profile of anemic women. We conducted a prospective study in the maternity department of the CHEIKH ZAYED Hospital Center in Nouakchott, during the period from 2015 to 2016. In addition, 400 pregnant women are interviewed, including $49.75 \%$ ( $n=199)$ with anemia, of which $34.17 \%$ were under 24 years of age, $63.31 \%$ are white Moors and $90.45 \%$ are at home. As a result, Statistical analyzes show a significant relationship, between certain socio-economic factors (Age, provenence area, ethnic origin, household size) and anemia.
\end{abstract}


Keywords: Anemia, pregnant woman, pregnancy, epidemiologic, Mauritania

\section{Resume}

L'anémie est l'un des problèmes de santé publique les plus répondus dans le monde, surtout chez les femmes enceintes. L'objectif de notre étude consiste à décrire le profil sociodémographique et épidémiologique des femmes anémiques. De ce fait, nous avons mené une étude prospective au sein du service de maternité du Centre Hospitalier CHEIKH ZAYED à Nouakchott, durant la période allant de 2015 à 2016. Notre échantillon est constitué de 400 femmes enceintes qui ont été interviewées lors de cette étude, dont 49,75\% $(\mathrm{n}=199)$ présentant des anémies, avec $34,17 \%$ ayant un âge inférieur à 24 ans, 63,31\% sont des maures blancs et 90,45\% sont au foyer. Les analyses statistiques montrent une relation significative entre certains facteurs socio-économiques (l'âge, le milieu de provenance, l'origine ethnique, la taille de ménage) et l'anémie.

Mots-Cles : Anémie, femme enceinte ,grossesse, épidémiologie, Mauritanie

\section{Introduction}

L'anémie est une chute de l'efficacité ou du nombre des globules rouges contenus dans le sang. L'anémie est l'une des modifications physiologiques de l'organisme féminin pendant la grossesse. Elle représente un problème majeur de santé publique dans les pays en voie de développement (Mac Phail P, 1992). La prévalence dans ces pays atteint environ $47 \%$ de la population totale contre $10 \%$ dans les pays développés (Van,Den,Broek, 2001). Cette anomalie est beaucoup plus fréquente en Asie Méridionale et en Afrique (Kalenga et al, 2003).

Selon l'Organisation Mondiale de la Santé (O.M.S), l'anémie nutritionnelle, chez une femme enceinte, est déclarée lorsque la teneur en hémoglobine dans le sang est inférieure à $11 \mathrm{~g} / \mathrm{dl}$ (OMS, 1992). Par ailleurs, en 1999, les travaux de Meda et al. au Baurkina Faso et ceux de Huddle et al au Melawi ont approuvé que la fréquence de l'anémie au cours de la grossesse est en nette progression, avec respectivement des prévalences de 66\% et 69\% (Meda et al., 1999 ; Huddle et al., 1999).

En outre, en Mauritanie, une étude menée en 1997 au Centre Hospitalier National (CHN) indique une prévalence de 53,1\% chez les femmes enceintes (BAIDY et al 1996). Depuis cette date, peu de travaux de recherches épidémiologiques se sont intéressés à l'anémie nutritionnelle des femmes enceintes à travers le territoire mauritanien. Devant cette situation, nous nous sommes fixé comme objectif de mener auprès de la population de Nouakchott (Capitale de la Mauritanie), une étude prospective transversale, 
afin d'évaluer la prévalence de l'anémie chez les femmes enceintes, et les facteurs socio-économiques associés

\section{Matériel et Méthodes}

\section{- Types, lieu et durée d'étude}

Il s'agit d'une étude prospective transversale, réalisée au Service de Maternité de l'Hôpital Cheikh Zayede à Nouakchott (CHCZ), durant la période allant du 08-2015 au 09-2016. Cette étude a porté sur 400 femmes enceintes, venant en consultation prénatale dans le CHCZ. Les données sont collectées sur la base d'un questionnaire anonyme comportant des questions sur l'appartenance ethnique, l'âge, le niveau d'instruction, la situation professionnelle de la femme et son mari, le type d'habitat, la présence d'eau et d'électricité.

\section{- Analyse statistique}

Les données recueillies sont saisies sur un support Excel, après filtration, elles sont transférées sur un support logiciel conçu pour 1'exploitation des données (SPSS. Version 22). Les analyses appliquées sont généralement des fréquences en pourcentage. Des analyses multiples, comme le test khi-deux d'indépendance ont été choisies pour la confirmation ou la recherche de liaison.

\section{Résultats et discussion}

\section{Caractéristiques sociodémographiques de l'échantillon}

Notre étude a porté sur un échantillon de 400 femmes enceintes, l'âge moyen de ces dernières est de 28,09 $\pm 0,32$ ans, avec un âge minimal de 14 ans et un âge maximal de 45 ans. La dispersion intra groupe représentée par le coefficient de variation (écart type/ moyenne) est de $22,43 \%$, ce qui qualifie notre échantillon comme assez homogène. La distribution s'est montrée gaussienne (coefficient d'asymétrie $=0,15$; coefficient d'aplatissement $=-0,56)$. Par ailleurs, 97,2\% $(n=389)$ de ces femmes enceintes proviennent du milieu urbain, représenté essentiellement par la ville de Nouakchott et $2,8 \%(\mathrm{n}=11)$ sont originaires des autres wilayas (provinces) du pays. Néanmoins, la répartition des femmes enceintes montre que $32,8 \%(n=131)$ ont un niveau scolaire primaire, contre 40,5\% $(n=162)$ ayant déclaré qu'elles ont atteint le stade secondaire et seulement $26,7 \%$ $(\mathrm{n}=107)$ ont atteint le niveau supérieur. Concernant la répartition selon la situation professionnelle des interviewées, les femmes aux foyers représentent 88,2\% ( $\mathrm{n}=353)$, par contre les femmes actives constituent $11,8 \%(\mathrm{n}=47)$ qui exercent soit en profession libre ou publique. 


\section{- $\quad$ Etude du profil épidémiologique de l'anémie au sein de notre échantillon}

Les femmes enceintes venant en consultation prénatale dans le CHCZ sont soumises à des analyses médicales par la Numération de la Formules Sanguines (NFS), pour déterminer le taux d'hémoglobine ( $\mathrm{Hb}: \mathrm{g} / \mathrm{dl})$. Les résultats de cette analyse montrent que 49,75\% $(n=199)$ des femmes enceintes de notre échantillon sont anémiques, par référence à la norme prescrite par l'OMS (taux d'hémoglobine inférieur à 11g/dl). Cette prévalence est inférieure à celle trouvée par BAIDY et al. (1996), au niveau de $\mathrm{CHN}$ à Nouakchott avec 53,1\%, et également proche de celle révélée par d'autres études effectuées dans des pays en voie de développent : $50 \%$ en Inde (Madan et al, 1990), 52\% au Nigeria (Fleming, 1990), et 61\% au Sénégal (Bayebi, 2013). En comparaison avec des prévalences rapportées dans les pays du Maghreb, celle de notre pays dépasse la prévalence trouvée au Maroc $(37,2 \%)$ et en Tunis (32,3\%), (Mohamed EL HIOUI, 2008) (Anémie en Tunisie 2002).

\section{- Etude de la liaison entre l'anémie et certains paramètres sociodémographiques}

Dans cette partie nous cherchons des liaisons éventuelles entre l'anémie et les variables suivantes : l'âge, le milieu de provenance, le niveau d'instruction, la situation professionnelle pour les femmes et leurs maris, l'origine ethnique, et la taille du ménage Les résultats de cette analyse sont consignés dans le tableau ci-dessous.

Tableau 1 : Etude de la liaison entre l'état anémique et certains facteurs sociodémographique

\begin{tabular}{|c|c|c|c|c|c|c|}
\hline Variable & Modalité & $\begin{array}{l}\text { Anémique } \\
(\mathrm{n}=199)\end{array}$ & $\begin{array}{c}\text { Non- } \\
\text { Anémiq } \\
\text { ue } \\
(n=201)\end{array}$ & $\begin{array}{l}\text { Total } \\
\text { (VPP) }\end{array}$ & $\begin{array}{l}\text { Khi- } \\
\text { deux }\end{array}$ & $\begin{array}{c}\mathrm{p} . \\
\text { value }\end{array}$ \\
\hline \multirow[t]{4}{*}{ Age } & $<24$ ans & $68(34,17 \%)$ & 52 & $\begin{array}{c}120 \\
(56,66 \%)\end{array}$ & \multirow[t]{4}{*}{3,766} & \multirow[t]{4}{*}{$\begin{array}{c}\mathbf{0 , 0 6 n} \\
\mathrm{s}\end{array}$} \\
\hline & $24<>28$ ans & $46(23,12 \%)$ & 47 & $\begin{array}{c}93(49,46 \% \\
)\end{array}$ & & \\
\hline & $28<>32$ ans & $41(20,60 \%)$ & 47 & $\begin{array}{c}88 \\
(46,60 \%) \\
\end{array}$ & & \\
\hline & $>32$ ans & $44(22,11 \%)$ & 55 & $\begin{array}{c}99 \\
(44,44 \%) \\
\end{array}$ & & \\
\hline \multirow{2}{*}{$\begin{array}{c}\text { Milieu de } \\
\text { provenanc } \\
\text { e }\end{array}$} & rural & $10(5,03 \%)$ & 1 & $11(90,90)$ & \multirow[t]{2}{*}{7,665} & \multirow[t]{2}{*}{$\mathbf{0 , 0 3 *}$} \\
\hline & urbain & $189(94,97 \%)$ & 200 & $\begin{array}{c}389 \\
(48,58 \%) \\
\end{array}$ & & \\
\hline $\begin{array}{l}\text { Niveau } \\
\text { scolaire }\end{array}$ & $\begin{array}{c}\text { Non } \\
\text { scolarisée et } \\
\text { niveau } \\
\text { primaire }\end{array}$ & $72(36,18 \%)$ & 59 & $\begin{array}{c}131 \\
(54,96 \%)\end{array}$ & 2,259 & $\begin{array}{c}\mathbf{0 , 1 5 n} \\
s\end{array}$ \\
\hline
\end{tabular}




\begin{tabular}{|c|c|c|c|c|c|c|}
\hline & $\begin{array}{c}\text { Niveau } \\
\text { secondaire }\end{array}$ & $78(39,20 \%)$ & 84 & $\begin{array}{c}162 \\
(48,14 \%)\end{array}$ & & \\
\hline & $\begin{array}{c}\text { Bac et } \\
\text { études } \\
\text { supérieur }\end{array}$ & $49(24,62 \%)$ & 58 & $\begin{array}{c}107 \\
(45,79 \%)\end{array}$ & & \\
\hline \multirow{2}{*}{$\begin{array}{c}\text { Situation } \\
\text { profession } \\
\text { nel pour la } \\
\text { femme }\end{array}$} & Active & $19(9,55 \%)$ & 28 & $47(40,42)$ & \multirow[t]{2}{*}{1,852} & \multirow[t]{2}{*}{$\begin{array}{c}\mathbf{0 , 1 7 2} \\
\mathrm{ns}\end{array}$} \\
\hline & Au foyer & $180(90,45 \%)$ & 173 & $\begin{array}{c}353 \\
(50,99 \%)\end{array}$ & & \\
\hline \multirow{3}{*}{$\begin{array}{c}\text { Situation } \\
\text { profession } \\
\text { nel pour } \\
\text { son mari }\end{array}$} & $\begin{array}{c}\text { Fonctionnair } \\
\text { e }\end{array}$ & $60(30,15 \%)$ & 75 & $\begin{array}{c}135 \\
(44,44 \%)\end{array}$ & \multirow[t]{3}{*}{2,436} & \multirow[t]{3}{*}{$\begin{array}{c}0,25 n \\
s\end{array}$} \\
\hline & $\begin{array}{c}\text { Activités } \\
\text { libres }\end{array}$ & $115(57,79 \%)$ & 102 & $\begin{array}{c}217 \\
(52,99 \%) \\
\end{array}$ & & \\
\hline & Autres & $24(12,06 \%)$ & 24 & $48(50 \%)$ & & \\
\hline \multirow[t]{3}{*}{$\begin{array}{l}\text { L'origine } \\
\text { ethnique }\end{array}$} & $\begin{array}{c}\text { Maure } \\
\text { Blanc }\end{array}$ & $126(63,31 \%)$ & 143 & $\begin{array}{c}269 \\
(46,84 \%)\end{array}$ & \multirow[t]{3}{*}{6,775} & \multirow[t]{3}{*}{$\mathbf{0 , 0 3 *}$} \\
\hline & Maure Noir & $61(30,66 \%)$ & 55 & $\begin{array}{c}116 \\
(52,58 \%)\end{array}$ & & \\
\hline & $\begin{array}{c}\text { Négro- } \\
\text { africaine }\end{array}$ & $12(6,03 \%)$ & 3 & $15(80 \%)$ & & \\
\hline \multirow[t]{2}{*}{$\begin{array}{l}\text { Taille de } \\
\text { la famille }\end{array}$} & $\leq 5$ & $95(47,74 \%)$ & 97 & $\begin{array}{c}192 \\
(49,47 \%)\end{array}$ & \multirow[t]{2}{*}{23,87} & \multirow[t]{2}{*}{$\mathbf{0 , 0 3 *}$} \\
\hline & $>5$ & $104(52,26 \%)$ & 104 & $\begin{array}{c}108 \\
(96,29 \%)\end{array}$ & & \\
\hline
\end{tabular}

ns : non significative, ${ }^{*}$ : différence significative, VPP : valeur prédictive positive ; ( ) : effectif

\section{$\checkmark \quad$ Répartition des femmes anémiques selon l'âge}

Notre étude révèle par ailleurs que les jeunes femmes de moins de 24 ans constituent un groupe à risque. Une telle observation est signalée par BAIDY et al. (1996) à Nouakchott. Ces résultats sont en accord avec ceux de Schol et al. (1994), qui ont montré que les femmes enceintes âgées de moins de 20 ans risquaient deux fois plus d'être anémiques que les femmes plus âgées. Par opposition, Prakash (1973) et Gujral et al. (1989), rapportent qu'au Québec, l'âge de la femme enceinte n'a pas d'influence significative sur la survenue de l'anémie.

\section{$\checkmark \quad$ Répartition des femmes anémique selon le milieu de provenance}

La répartition des femmes en état d'anémie selon le milieu de provenance est présentée dans le tableau 1. Les femmes qui souffrent d'une anémie sont en majorité d'origine urbaine $(n=189)$, ce qui représente $95 \%$ de cas malades contre uniquement $5 \%$ provenant du milieu rural $(n=10)$. Contrairement, l'enquête sur les causes et mesures d'intervention de l'anémie des femmes en Tunisie (2002), qui a montré que la distribution interrégionale de l'anémie chez les femmes enceintes est homogène, la prévalence de l'anémie sévère est modérée soit $2,7 \%$ dans les régions du 
Grand Tunis et 2,5\% pour les régions du Sud Ouest, sans noter une différence significative entre les deux. Le test khi-deux montre une liaison significative entre l'état de santé et le milieu de provenance (khi-deux = $7,665 ; \mathrm{p}<0,03)$.

\section{$\checkmark \quad$ Répartition des femmes anémiques selon le niveau d'instruction \\ L'objectif de l'étude par rapport au niveau d'instruction est de valoriser, la capacité des femmes à répondre correctement aux questions posées et à comprendre tous message concernant une éducation sanitaire ultérieure. $36,18 \%$ des femmes interrogées ont déclaré avoir un niveau primaire ; 39,20\% ont le niveau secondaire, et seulement 24,62 ont atteint le niveau supérieure. Aucune relation significative $(\mathrm{p}<0,15)$ n'a été observée entre le niveau d'éducation des femmes et l'affection par l'anémie. Ceci revient au fait que la plupart des femmes enquêtées sont analphabètes ou ayant juste un niveau secondaire. Ce résultat est similaire à une étude réalisé au Maroc (Sellam et Bour, 2014). Cependant, une étude réalisée dans la région de Kénitra, a montré que la prévalence de l'anémie diminue avec le niveau d'études des femmes (Aboussaleh et al., 2009). \\ $\checkmark \quad$ Répartition des femmes anémiques selon leur situation professionnelle}

La répartition selon la situation professionnelle montre que la majorité des femmes enquêtées sont sans profession. Parmi 199 femmes anémiques, $90 \%(\mathrm{n}=180)$ sont des femmes au foyer alors que les femmes actives représentent 19 cas soit (10\%), Le test khi-deux d'indépendance ne montre aucune association entre ces deux facteurs (khi-deux $=1,852$; $\mathrm{p}<0,172$ ). Nos résultats sont proches de ceux de TOUNKARA (2011) qui réfère le taux de femmes anémiques sans profession au Mali à 87,6\% de la population touchée par l'anémie. Par contre à Naplouse (AMANI, 2007), l'étude de la prévalence de l'anémie en fonction de la situation professionnelle marque un taux élevé $26,7 \%$ dans le group de femmes enceintes travailleuses par rapport aux femmes ménagères qui est de $21,4 \%$, cela pourrait être du à l'effort au travail et au manque de repos ainsi que manque de nutriments nécessaires à leurs période gravidique.

\section{$\checkmark \quad$ Répartition des femmes anémiques selon l'origine ethnique}

En Mauritanie il y a trois types d'origine ethnique. Cette étude montre que, 126 femmes anémiques soit $(63 \%)$ sont des maures blancs (maure $=$ mauritanien), alors que 61 femmes soit $(31 \%)$ des maures noirs et 12 femmes soit $(6 \%)$ sont des négro-africaines. Cependant, la valeur prédictive positive est beaucoup plus importante chez les femmes négroafricaines $(80 \%)$ que les autres ethnies. Cet écart important est imputé aux 
problèmes de représentabilité du faite que la majorité des femmes visitant l'hôpital sont d'origine ethnique maure blanc. L'analyse statistique montre une relation significative $(\mathrm{p}=0,03)$ entre l'anémie et l'origine ethnique. Ce résultat confirme celui d'autres auteurs (TOUNKARA, 2011).

\section{$\checkmark \quad$ Répartition des femmes anémiques selon la taille de la famille}

La répartition des femmes enceintes selon la taille du ménage et l'anémie, supporte l'hypothèse d'une certaine augmentation de la prévalence de l'anémie chez les femmes provenant de grands ménages, ce qui semble logique, puisque les plus grands foyers ont moins de moyens. Le test khideux montre une liaison significative entre l'anémie et la taille du ménage (khi-deux $=23,875 ; \mathrm{p}<0,03$ ), contrairement aux travaux menés par le ministère de la santé en Tunisie (2002), et les résultats d'une étude réalisé au Maroc (Sellam et Bour, 2014). Concernent la situation professionnelle des conjoins, la majorité ont des activités libres $57,79 \%$, alors que les fonctionnaires représentent $30,15 \%$, Cette situation est, fort probablement, liée au niveau socio-économique du ménage.

\section{Conclusion}

Malgré l'amélioration des conditions du niveau de vie ces dernières décennies, l'anémie chez les femmes enceintes reste un problème majeur de santé publique en Mauritanie. Ces résultats montrent un niveau de prévalence de l'anémie très élevée dans la région de Nouakchott. La couverture de l'ensemble du district sanitaire de la République Islamique de Mauritanie (RIM) par cette étude donnerait une meilleure cartographie du niveau de la prévalence de ce problème de santé chez les femmes enceinte et de l'évolution de la prise en charge. La prévention de l'anémie chez ces dernière doit être liée à une bonne éducation sanitaire et surtout nutritionnelle pour chaque femmes en âge de procréer.

\section{Remerciements}

Nos remerciements à Mr. chef service de gynécologue-obstétrique au l'Hôpital Cheikhe Zayed,(CHCZ) en Mauritanie. Je lui suis reconnaissant de m'avoir accueilli et conseillé, plusieurs fois dans son bureau et même sans rendez-vous, lors des différentes étapes de cette recherche. Je remercié aussi le personnels de santé de même service qui ont contribués de près ou de loin à cette étude et pour leurs aides. Nos remerciements également au Mr. Chef de département sciences de la vie, directeur de cette recherche, et à tout 1'équipe du laboratoire de Biochimie, Biotechnologie, Environnement et Santé, département sciences de la vie Faculté de Sciences Université IBN TOFAIL, Kénitra, Maroc. 


\section{References:}

1. Aboussaleh Y, Farsi, M, El Hioui M, Ahami, A., 2009. Transition nutritionnelle au Maroc: Coexistence de l'anémie et de l'obésité chez les femmes au Nord Ouest marocain. Antropo, 19, 67-74. www.didac.ehu.es/antropo

2. A.Demmouche, S. Moulessehoul Prévalence de l'anémie ferriprive au cours de la grossesse dans la wilaya de Sidi Bel Abbes (ouest de l'Algérie) Demmouche et al. 2010. Antropo, 21, 39-48: 46. www.didac.ehu.es/antropo

3. Amani Waleed Mahmoud Abu-hasira «Iron Deficiency Anemia among Pregnant Women in Nablus District; Prevalence, Knowledge, Attitude and Practices» An-Najah National University Faculty of Graduate Studies Nablus, Palestine, 2007

4. Anémie en Tunisie: Causes et Mesures d'Intervention, Ministère de la santé $2002: 50,71$

5. B.LO. BAIDY, Y. KONE ET LY. BASSIROU,Anémie nutritionnelle de la grossesse à Nouakchott Médecine d'Afrique Noire : 1996, 43 (6), 356

6. Bayebi BADO, Analyse des déterminants socio-économiques de l'anémie ferriprive chez les femmes enceintes au Sénégal : cas de la région de Dakar 2012-2013. 2

7. Fleming A.F., 1990, Malaria deficiencies of iron and folate, and anaemia in pregnancy in the Guinea Savanna of Nigeria. In: Aspect actuel des carences en fer et folates dans le monde. S. Hercberg, P. Galan, H. Dupin. eds. Ed. Colloque INSERM. 197: 71-74

8. Huddle JM, Gibson RS, Gullinan TR. The impact of malarial infection and diet on the anaemia status of rural pregnant Malawian women. Eur J Clin Nutr 1999; 53: 792-801

9. Mac Phail P, Bothwell TH. Th prevalence and causes of nutritional iron deficiency anemia. In: Fomon SJ, zlotkin S, eds. Nutritional anemia. New York: raven press 1992: 1-12

10. Meda N., Dao Y., Touré B., Yamego B. et al., 1999, Evaluer l'anémie maternelle sévère et ses conséquences: la valeur d'un simple examen de la coloration des conjonctives palpébrales. Cahier santé, volume 9, Numéro 1: 7- 11.

11. Med N . Mandel Brot L, Cartaux M,Dao B, Ouangre A, Dabis F. Anaemia during pregnancy in Burkina Faso,west Africa : prevalence and associated factors. DITRAME Study Grou.Bull World Health Organ 1999; 77; 916-22

12. M.K.Kalenga,et colloborateurs. Etude de l'anémie chez les femmes encentes et les femmes allaitantes de Lubumbashi ( Repeblique 
Démographique du Congo), J Gynecol obstet Biol Reprod/ Volum $32, n^{\circ} 7,2003 ; 648$

13. Mohamed El HIOUI, Etude congnitivo-comportementale, neuropsychologique et nutritionnelle chez les consultants adultes et les écoliers anémiques de la région rurale de Kénitra $2008: 13$

14. MoryDant TOUNKARA, Anémie sévère sur grossesse a l'hôpital fousseyni daou de kayes, $2011: 46,48,74$

15. Organisation Mondiale De La Santé, 1992, Les anémies nutritionnelles. Série de rapports techniques. $N^{\circ} 503$ Genève. pp 6

16. Sellam et Bour, 2014. Anémie chez les femmes en âge de procréer au Maroc (Préfecture d'Oujda-Angad) Antropo, 32, 35-44. www.didac.ehu.es/antropo

17. Van Den Broek N. Anaemia in Pregnancy in sub-saharan countries. Eur J Obestet Gyn RP 2001 ; 96 4-6 